\title{
Real-gas effect accounted for by the Principle of Extended Corresponding States in modeling supersonic kerosene jet
}

\author{
Ye Wang ${ }^{1}$ Wei Yao ${ }^{2}$ Xuejun Fan ${ }^{3}$ \\ a. State Key Laboratory of High Temperature Gas Dynamics, Institute of Mechanics, CAS, No.15 Beisihuanxi \\ Road, Beijing 100190, China
}

b. School of Engineering Science, University of Chinese Academy of Sciences, Beijing 100049, China

Supersonic kerosene jet was computationally investigated by Large Eddy Simulation (LES) combined with the principle of Extended Corresponding States (ECS) applied to model real-gas effect by characterizing the pressure-temperature-density behavior of real kerosene. Two cases including real-gas effect considered jet using the ECS principle and real-gas effect neglected jet using ideal gas law were both set to comparatively reveal the flow evolution and development. The modeling results achieved good agreements with the measured near nozzle shock structures in experiments. Furthermore, qualitative and quantitative analyses of flow properties like shock structures, aerodynamic fields, and mixing characteristics were conducted to display the influence of real-gas effect on the kerosene jet properties. The results show that the Mach disk is smaller and closer to nozzle exit in real-gas effect considered jet. And its temperature, pressure and density are higher, but the Mach number is lower at the nozzle exit and upstream of the normal shock. In addition, the diffusion physics is very similar in the two jets, but a little bit shorter potential core and larger diffusion area appear in real-gas effect considered jet.

\section{Nomenclature}

$P \quad=$ pressure

$\rho \quad=$ density

$T=$ temperature

$u_{i} \quad=$ velocity in $x_{i}$ direction

$\tau_{i j} \quad=$ viscous stress tensor

$h_{s} \quad=$ sensible enthalpy

$q_{i} \quad=$ heat flux tensor

$Y_{k} \quad=$ mass fraction of species $\mathrm{k}$

$D_{k} \quad=$ equivalent mass diffusion coefficient

$H_{i} \quad=$ energy fluxes in $\mathrm{x}_{\mathrm{i}}$ direction

$\Phi_{k, j} \quad=$ species fluxes in $\mathrm{x}_{\mathrm{j}}$ direction of species $\mathrm{k}$

$\boldsymbol{U}=$ velocity vector

$\mathrm{Ma} \quad=$ Mach number

Re $\quad=$ Reynolds number

$H_{m} \quad=$ height of Mach disk

$D_{m} \quad=$ width of Mach disk

$\theta_{m} \quad=$ expansion angle of Mach disk

$\alpha_{m} \quad=$ shock reflection angle

$D \quad=$ diameter of the nozzle exit

$A=$ area

$S=$ strain rate tensor

$\Omega \quad=$ rotation rate tensor

$Q \quad=\quad \mathrm{Q}$ - criterion

\footnotetext{
${ }^{1}$ Master Student, Institute of Mechanics (CAS), wangye@imech.ac.cn.

${ }^{2}$ Associate Professor, Institute of Mechanics (CAS), weiyao@imech.ac.cn, AIAA member (Corresponding author).

${ }^{3}$ Professor, Institute of Mechanics (CAS), xfan@imech.ac.cn, AIAA lifetime member (Corresponding author).
} 
$x, y, z=$ Cartesian coordinates

\begin{tabular}{ll}
\multicolumn{2}{l}{ Superscripts } \\
- & $=$ cell average \\
$\sim$ & $=$ Favre average \\
sgs & $=$ subgrid-scale
\end{tabular}

Subscripts

$\infty \quad=$ ambient condition

$0=$ injection condition

$e \quad=$ condition at nozzle exit

$u=$ condition at upstream of the normal shock

$m \quad$ = parameter of Mach disk

\section{Introduction}

$\mathrm{I}_{\mathrm{b}}^{\mathrm{n}}$ modeling hydrocarbon fuel jets, accurate descriptions of the gas state and thermophysical properties are important because they significantly influence the jet breakup and the subsequent mixing. However, the gas density is usually simulated simply by ideal gas law, and the thermophysical properties are accounted for by the values in the gas phase. Since both molecular size and inter-molecular attractions are neglected in the ideal gas law, it is not accurate anymore for non-monatomic gases at high pressures and low temperatures. In scramjet combustors, the kerosene is usually heated to supercritical status before issued into the domain. For supercritical kerosene, the temperature and pressure are above $630 \mathrm{~K}$ and above $2.4 \mathrm{MPa}$ respectively [1]. Due to the initial expansion of the jet, the kerosene temperature can become much lower than its boiling point, so that both the liquid and the vapor phases may coexist. Under such high pressures and potential low temperatures, the real-gas effect should be accounted for, for example by the principle of Extended Corresponding States (ECS) [2], to accurately characterize the jet penetration and breakup, which are important for the following fuel mixing and combustion.

Little research has been conducted to examine the influence of real-gas effect on the jet breakup and mixing. However, some equations of state that account for real-gas effects are used in previous studies. Petit et al. [3] simulated the injection of supercritical nitrogen into a warm nitrogen environment, in which Soave-Redlich-Kwong (SRK) and Peng-Robinson (PR) equation of state (EoS) were both used. It was shown that they both do well in predicting the density and temperature in the far field. Oefelein [4] demonstrated that both the SRK- and PR-EoS can give accurate results over the applied range of pressure, temperature, and states of the mixture when they were used with ECS. Furthermore, it was recommended that SRK-EoS was much more exact for low temperatures, but PR-EoS performed better in flow fields with intense heat release. Hamzehloo et al. [5] and Bonelli et al. [6] found evident differences between the predictions on Mach number and pressure by the ideal gas law, the Van-Der-Waals EoS, and the RedlichKwong EoS by simulating a hydrogen jet at high injection pressure. The above cubic EoSs are computationally efficient but less accurate, especially for mixtures at near-critical conditions than Benedict-Webb-Rubin (BWR) EoS [7]. A 32-term BWR EoS was used to predict the pressure-volume-temperature behavior of liquid-phase, saturatedvapor, and gas-mixture properties in the vicinity of the critical point and achieved a good result [4].

However, these equations of state are rarely used alone for hydrocarbon fuels, especially kerosene, because of the huge number of their species which need heavy workload to standardize parameters in the formulas. Previous researchers $[1,2,8]$ have shown that the principle of extended corresponding states (ECS) [2] can accurately describe the properties of supercritical kerosene. ECS principle is based on the fact that the state curves of different fluids are similar, and is used to obtain an unknown mixture state from the known through scaling state variables and similarity transformations [1,9]. Ely and Hanley [10,11] developed the ECS methods to conduct viscosity and thermal conductivity. Takahashi [12] proposed the corresponding-state methodology to obtain mass diffusion coefficients and thermal diffusion coefficients. Oefelein [13] simulated the shear-coaxial liquid-oxygen-hydrogen jet flame at supercritical pressure, where the ECS model was used with a cubic equation of state to evaluate the pressure-volumetemperature behavior.

In the present study, ECS principle is used to model the real pressure-temperature-density behavior in the threedimensional large eddy simulation (LES) of supersonic kerosene jet with an exit Reynolds number around $8 \times 10^{5}$. And the jet penetration, instability, breakup, diffusion, mixing characteristics and near nozzle shock structures are discussed. In addition, the flow parameters of real-gas effect considered jet are compared with results in real-gas effect neglected jet using ideal gas law as well as the experimental results conducted by the same authors' group. 


\section{Numerical methodology}

\section{A. Fuel surrogate}

A lot of research on physical and chemical properties and supersonic combustion characteristics of Daqing RP-3 aviation kerosene has been carried out by Institute of Mechanics, CAS [1, 14-19]. The chemical composition of Daqing RP-3 is extremely complicated because it has thousands of species, such as straight-chain alkanes, naphthenes and aromatic compounds, which changes with the origins, manufactures and many other reasons. In order to maintain the consistency of the study objects and the reproducibility of the results and to simplify the numerical simulation, it is vital to find out an appropriate fuel surrogate model to simulate the thermophysical properties as well as the injection, mixing and combustion of the complex real fuel. There have been some attempts at research on fuel surrogate. Daniau et al. [20] replaced US JP-7 aviation kerosene directly with n-dodecane for studies of cracking and heat transfer. Dagaut [21] proposed a three-component fuel surrogate model for French TR0 aviation kerosene, which consisted of $74 \%$ n-decane $\left(\mathrm{C}_{10} \mathrm{H}_{22}\right), 11 \%$ propylcyclohexane $\left(\mathrm{C}_{9} \mathrm{H}_{18}\right)$ and $15 \%$ n-propylbenzene $\left(\mathrm{C}_{9} \mathrm{H}_{12}\right)$ in mole fraction. As Daqing RP-3 is more similar to TR0 in terms of density and composition, Fan et al. [1] selected a surrogate model composed of $49 \%$ n-decane $\left(\mathrm{C}_{10} \mathrm{H}_{22}\right), 44 \%$ 1, 3, 5-trimethylcyclohexane $\left(\mathrm{C}_{9} \mathrm{H}_{18}\right)$ and $7 \%$ n-propylbenzene $\left(\mathrm{C}_{9} \mathrm{H}_{12}\right)$ in mole fraction, based on the surrogate model presented by Dagaut [21], to simulate the thermophysical and transport properties of Daqing RP-3 aviation kerosene. In this study, a widely used surrogate of Gas-to-Liquid (GtL) aviation kerosene proposed by Dagaut et al. [22] is selected, consisting of $57.7 \%$ n-decane $\left(\mathrm{C}_{10} \mathrm{H}_{22}\right)$, 33.2\% 2,2,4trimethylpentane $\left(\mathrm{C}_{8} \mathrm{H}_{18}\right)$ and $9.1 \%$ n-propylcyclohexane $\left(\mathrm{C}_{9} \mathrm{H}_{18}\right)$ in mole fraction as shown in Table 1. Furthermore, it has been demonstrated that this surrogate showed a behavior which is similar to many jet fuels, ideally for predicting both chemical and physical properties [22].

Table 1. Three-component fuel surrogate proposed by Dagaut et al. [22].

\begin{tabular}{ccc}
\hline Compound & Formula & Mole fraction, \% \\
\hline N-decane & $\mathrm{C}_{10} \mathrm{H}_{22}$ & 57.7 \\
\hline 2,2,4-trimethylpentane & $\mathrm{C}_{8} \mathrm{H}_{18}$ & 33.2 \\
\hline N-propylcyclohexane & $\mathrm{C}_{9} \mathrm{H}_{18}$ & 9.1 \\
\hline
\end{tabular}

\section{B. Numerical details}

LES is used to characterize an unsteady, compressible, multi-component and non-reacting flow in present study, so the three-dimensional, filtered Navier-Stokes equations are applied to govern it, as in Eq. (1) - (4).

$$
\begin{gathered}
\frac{\partial \bar{\rho}}{\partial t}+\frac{\partial \bar{\rho} \widetilde{u}_{i}}{\partial x_{i}}=0 \\
\frac{\partial \bar{\rho} \widetilde{u}_{i}}{\partial t}+\frac{\partial \bar{\rho} \widetilde{u}_{i} \widetilde{u}_{j}}{\partial x_{j}}=-\frac{\partial \bar{p}}{\partial x_{i}}+\frac{\partial \tilde{\tau}_{i j}}{\partial x_{j}}-\frac{\partial \tau_{i j}^{s g s}}{\partial x_{j}} \\
\frac{\partial \widetilde{\rho} \widetilde{h}_{s}}{\partial t}+\frac{\partial \bar{\rho} \widetilde{u}_{j} \widetilde{h}_{s}}{\partial x_{j}}=\frac{\partial \bar{p}}{\partial t}+\frac{\partial \widetilde{u}_{j} \tilde{\tau}_{i j}}{\partial x_{i}}-\frac{\partial \bar{q}_{i}}{\partial x_{i}}-\frac{\partial H_{i}^{s g s}}{\partial x_{i}} \\
\frac{\partial \widetilde{\rho} \widetilde{Y}_{k}}{\partial t}+\frac{\partial \bar{\rho} \widetilde{u}_{j} \tilde{Y}_{k}}{\partial x_{j}}=\frac{\partial}{\partial x_{j}}\left(\bar{\rho} \bar{D}_{k} \frac{\partial \tilde{Y}_{k}}{\partial x_{j}}\right)-\frac{\partial \Phi_{k, j}^{s g s}}{\partial x_{j}}
\end{gathered}
$$

where '_' means cell average, ' $\sim$ ' means Favre average. $\rho, \mathrm{p}, \mathrm{u}_{i}$ and $\tau_{i j}$ are dynamic parameters, the density, the pressure, the velocity in $\mathrm{x}_{\mathrm{i}}$ direction and the viscous stress tensor, respectively. $h_{s}, q_{i}$ are both thermodynamic variables, the sensible enthalpy per unit mass and the heat flux tensor. $Y_{k}$ and $\mathrm{D}_{k}$ are the parameters of component transport, the mass fraction and the equivalent mass diffusion coefficient. All terms with superscript sgs denote subgrid-scale (SGS) quantities and they are modeled based on the eddy diffusivity assumption. In particular, the SGS stress, $\tau_{i j}^{s g s}=\bar{\rho}\left(\widetilde{u_{l} u_{J}}-{\widetilde{u_{l}}}_{u_{J}}\right)$, is modeled with the one-equation turbulent kinetic energy (one-eq K) [23], the SGS energy fluxes $H_{i}^{s g s}$ and SGS species fluxes $\Phi_{k, j}^{s g s}$ are modeled by using linearization method and introducing turbulent Prandtl number and Schmidt number.

Notably, methods to model the pressure-temperature-density behaviors of the two different cases, real-gas effect considered jet and neglected jet, are quite different. In detail, the pressure-temperature-density behavior of the realgas effect neglected case is predicted by ideal gas law. The real-gas effect for considered jet is accounted for by the principle of Extended Corresponding States. To be specific, the real density is computed by calling the database of 
hydrocarbon fuel components from NIST Supertrapp database and software package [2], and then the real pressure can be calculated by multiplying a similar transformation coefficient.

The above equations are solved by a density-based finite volume supersonic compressible flow solver, AstroFoam [24-28], which is developed based on the standard compressible flow solver rhoCentralFoam distributed with C++ open-source CFD package OpenFOAM V3.0.1 [29] mainly through adding the features of multi-species transport and multi-component reaction. Some CFD libraries are coupled into AstroFoam to characterize this problem, NISTJANAF thermo-physical and transport database [30] is developed to calculate the multi-species thermodynamic and transport properties; NIST Supertrapp software [2] package is used to get the real density and pressure with the principle of extended corresponding states; The semi-discrete central Kurganov-Tadmor (KT) scheme [31] is applied to evaluate convective fluxes for shock capturing and turbulence resolving; The low-dissipation scale-selective discretization (SSD) scheme [32] is adopted to reconstruct the primitive values at faces.

\section{Computational setup}

An appropriate flow region should be carefully selected for the effective and precise numerical simulation of the present supersonic jet. Figure 1 illustrates the computational domain with specific geometries $260 \mathrm{~mm}$, 90mm and $90 \mathrm{~mm}$ in $\mathrm{x}-, \mathrm{y}$ - and $\mathrm{z}$-directions, respectively. The starting jet is expected to emerge from the convergent-divergent nozzle with $28.8 \mathrm{~mm}$ total height and $3.164 \mathrm{~mm}$ exit diameter, the three-dimensional geometric model of the nozzle is shown in Figure 2. In addition, the same nozzle has been used to conduct several corresponding experiments by the same authors' group.

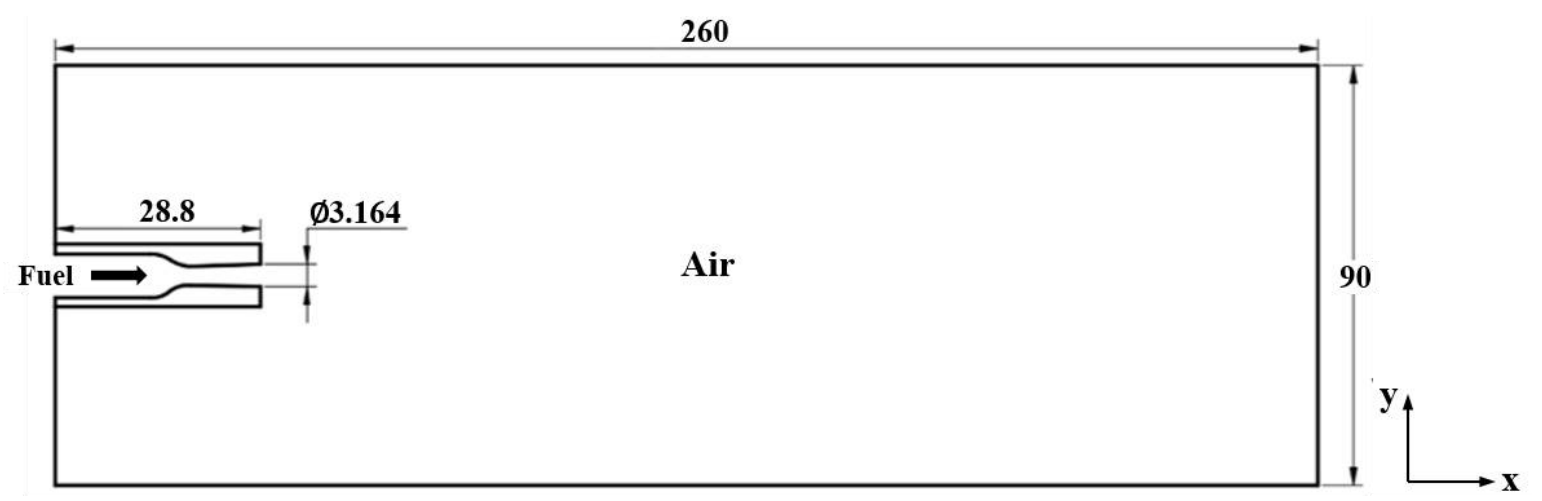

Figure 1. Schematic of the computational model at cross streamwise view

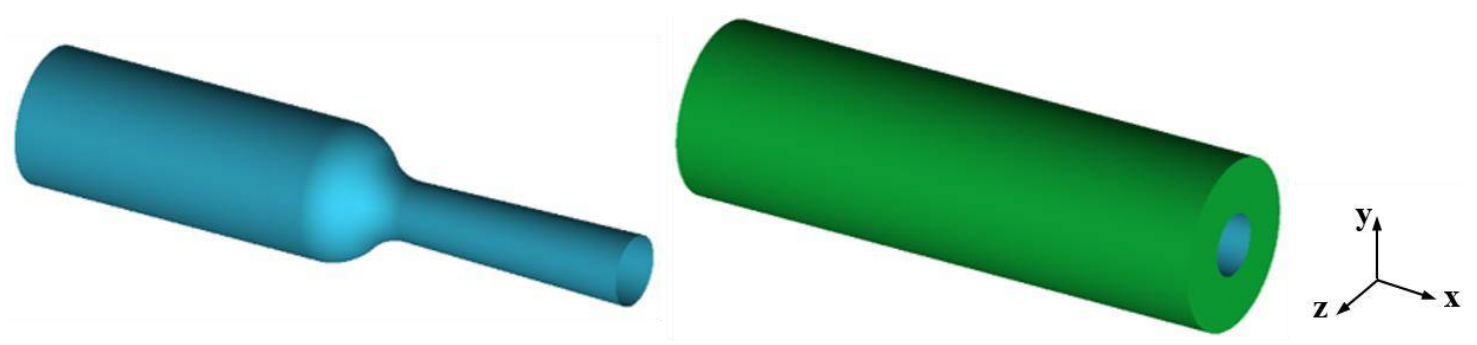

Figure 2. Geometric model of the nozzle

Figure 3 displays the hexahedral structured grid of the computational model. To make the results of LES better, the spatial resolution of the grid should be rather high. The grid inside and around the nozzle has been refined to capture the drastic changes in the flow. Besides, coarse cell sizes in the far fields are used to avoid wave reflections. The total cell number in the mesh is 9.7 million.

In present study, same starting conditions are applied to the two cases. Surrogate kerosene with static pressure $2 \mathrm{MPa}$ and static temperature $850 \mathrm{~K}$ is injected into quiescent air consisting of $76.699 \%$ nitrogen and $23.301 \%$ oxygen in mass fraction. The ambient air has uniform distribution of flow parameters, such as pressure $\mathrm{P}_{\infty}=101,325 \mathrm{~Pa}$, temperature $T_{\infty}=298 \mathrm{~K}$, velocity $\mathbf{U}_{\infty}=(0,0,0) \mathrm{m} / \mathrm{s}$ and density $\rho_{\infty}=1.17 \mathrm{~kg} / \mathrm{m}^{3}$. Moreover, the kerosene jets are issued 
from the nozzle exit and enter into air at Mach number 1.43 and 1.50 and Reynolds number $8.13 \times 10^{5}$ and $8.06 \times 10^{5}$ in real-gas effect considered and neglected jet severally. Some details of flow conditions in real-gas effect considered jet are listed in Table 2. The flows at inlet of nozzle are subsonic, thus the inlet conditions of velocity are zero-gradient. Besides, no-slip, adiabatic wall conditions are enforced on the surface of the nozzle and open boundary conditions are used at the far fields and the domain outlet.

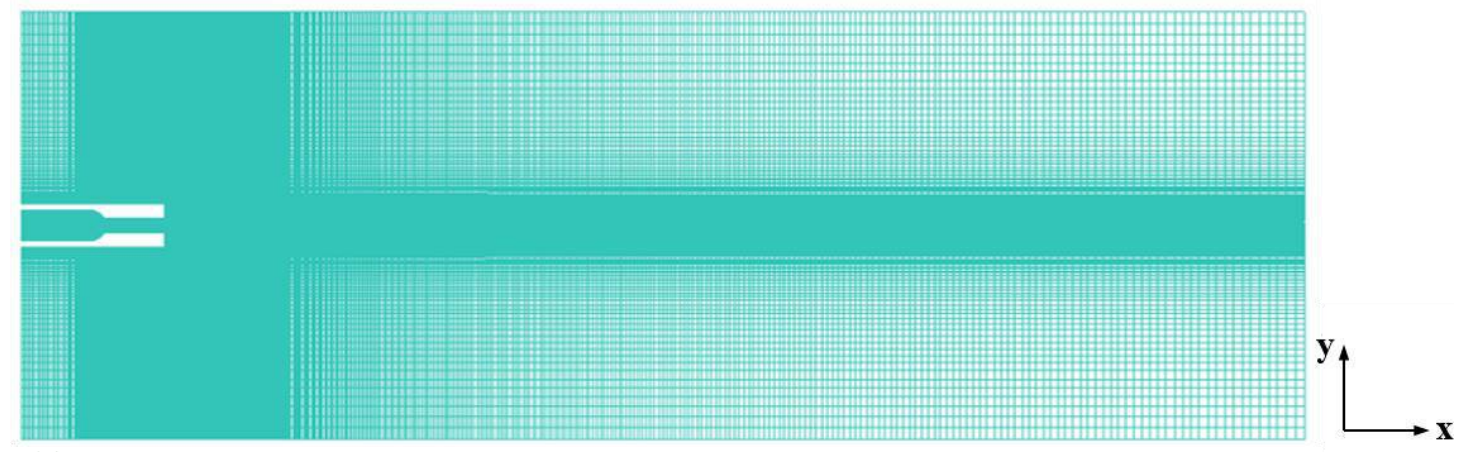

(a)

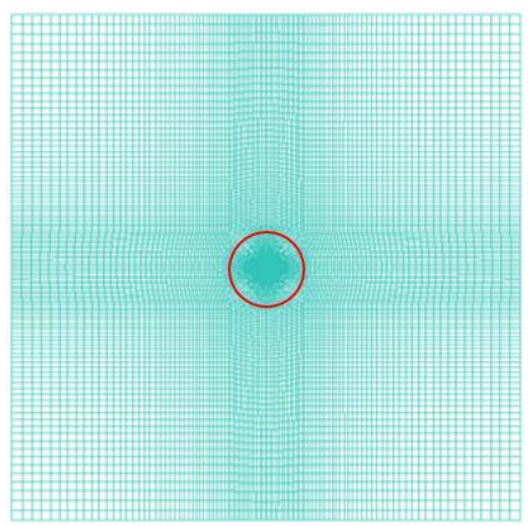

(b)

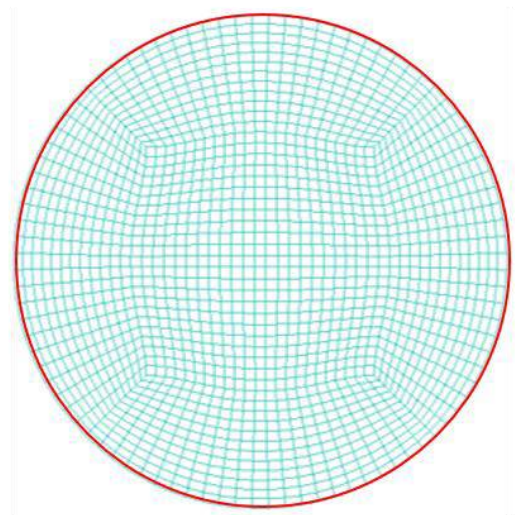

(c)

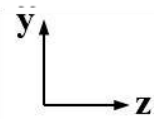

Figure 3. Computational grid: (a) view of streamwise; (b) top view at outlet; (c) partially enlarged view at the exit of nozzle

Table 2. Flow parameters of real-gas effect considered jet

\begin{tabular}{lc}
\hline Parameter & Value \\
\hline Nozzle pressure ratio (NPR), $\mathrm{P}_{0} / \mathrm{P}_{\infty}$ & 20 \\
Nozzle temperature ratio (NTR), $\mathrm{T}_{0} / \mathrm{T}_{\infty}$ & 2.85 \\
Mach number at nozzle exit, $\mathrm{Ma} e$ & 1.43 \\
Reynolds number at nozzle exit, $\mathrm{Re}_{\mathrm{e}}$ & $8.13 \times 10^{5}$ \\
Mach number at upstream of the normal shock, Mau & 2.98 \\
\hline
\end{tabular}

\section{Results and discussion}

\section{A. Near nozzle aerodynamic fields}

The computational results present a highly under-expanded jet flow field. The shock structures like Mach disk, reflected shock, barrel shock, triple points and slip lines in near fields are clearly captured. The mean near-field wave structures which are characterized by density gradient $\left(\log _{10}|\nabla \rho|\right)$ of real-gas effect considered jet using the ECS principle are shown in Figure 4, and the comparisons with experimental schlieren photography conducted by the 
authors' group and numerical schlieren of real-gas neglected jet using ideal gas law are demonstrated here. Furthermore, the shock structure parameters obtained from flow fields with real-gas effect, such as the height $\mathrm{H}_{\mathrm{m}}$ and the width $D_{m}$ of Mach disk, the expansion angle $\theta_{m}$ and the shock reflection angle $\alpha_{m}$, show good agreements with experimental results under injection conditions of nozzle pressure ratio (NPR) $=35$ and nozzle temperature ratio (NTR) $=2.52$. The details of these parameters are listed in Table 3. What is noteworthy is that the NPR and NTR have been decreased to 20 and increased to 2.85 respectively in the modeling to match with the experimental results. The measuring points of pressure and temperature in the referenced experiment are at the upstream of the kerosene tank, thus total pressure loss and heating phenomena should occur when kerosene flows through valves and pipes with friction. However, Figure 4 and Table 3 also indicate that the width $D_{m}$ of Mach disk in LES is a little bit greater than that in experiment for the lack of the consideration of pyrolytic reaction triggered by high temperature in LES. Pyrolytic reaction turns kerosene into a mixture of small molecules and the resulting smaller gas constant exactly corresponds with higher sound speed and lower Mach number, which leads to a narrower Mach disk. Another reason is the inaccurate fuel surrogate model. As for the comparison between real-gas effect considered and unconsidered jets, it is found that a lower and narrower Mach disk in real-gas effect considered jet, and the expansion angle and the shock reflection angle are almost equal severally. And the size of Mach disk in LES of real-gas neglected kerosene jet is similar to data of ideal gas conducted by Crist et al. [33].

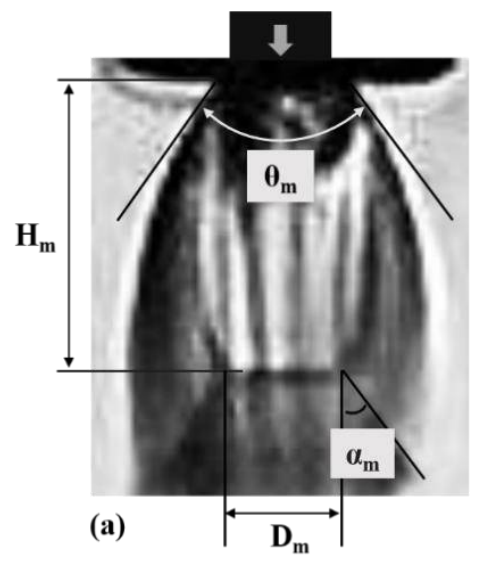

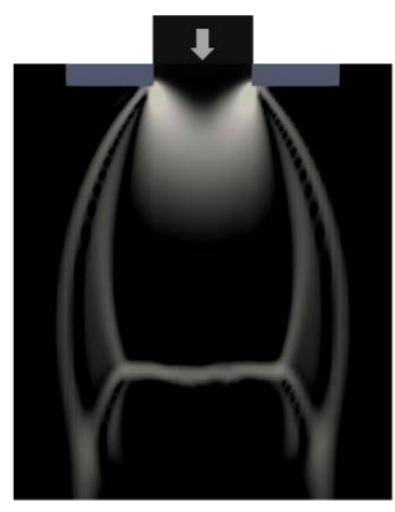

(b)

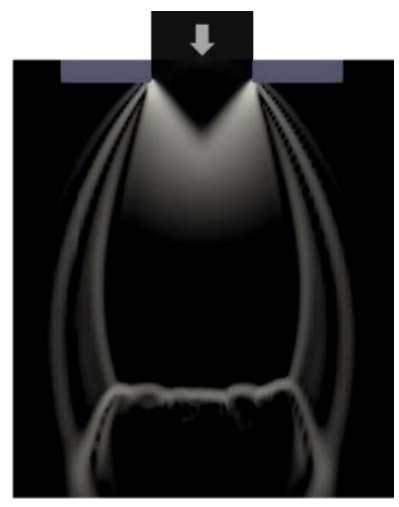

(c)

Figure 4. Comparison of near-field mean shock structures characterized by density gradient $\left(\log _{10}|\nabla \rho|\right)$ among experimental and LES's results: (a) experiment; (b) LES with ECS principle; (c) LES with ideal gas law

Table 3. Parameters of near-field shock structures (units: length in $\mathbf{m m}$, angle in ${ }^{\circ}$ )

\begin{tabular}{ccccc}
\hline Method & $\mathbf{H}_{\mathbf{m}}$ & $\mathbf{D}_{\mathbf{m}}$ & $\boldsymbol{\theta}_{\mathbf{m}}$ & $\boldsymbol{\alpha}_{\mathbf{m}}$ \\
\hline Experiment & 9.09 & 3.76 & 28.3 & 63.2 \\
\hline LES with ECS principle & 8.83 & 4.76 & 27.4 & 69.4 \\
\hline LES with ideal gas law & 9.64 & 5.38 & 27.9 & 70.9 \\
\hline Data of $\mathrm{N}_{2}$ by Crist et al. [33] & 9.13 & 5.00 & -- & -- \\
\hline
\end{tabular}

Figure 5 presents the comparisons of near-fields mean profiles of temperature, pressure, density and Mach number between real-gas effects considered jet (use ECS principle) and neglected jet (use ideal gas law) along the jet centerline. It is necessary to note that all flow parameters are nondimensionalized by injection parameters with subscript 0 , thereinto $\mathrm{P}_{0}=2 \mathrm{MPa}, \mathrm{T}_{0}=850 \mathrm{~K}, \rho_{0}=40.52 \mathrm{~kg} / \mathrm{m}^{3}$. It is shown clearly in Figure 5 that the two kerosene jets both accelerate to supersonic through the convergent-divergent nozzle, and a region with low temperature, low density, low pressure and high speed ( $\mathrm{Ma}_{\max }$ 3.0) forms before Mach disk because of the rapidly expansion of kerosene jet. Consequently, kerosene jets turn to subsonic after being compressed by Mach disk, and the temperature, density, and pressure also sharply increase. In more detail, the temperature, pressure and density decrease in the nozzle, and they decrease to larger values in real-gas effect considered jet than another at the nozzle exit. However, the variation tendency of Mach number is opposite. The Mach numbers at nozzle exit and upstream of the normal shock are lower in real-gas effect considered jet. The jumps of temperature, pressure and density and the drop of speed occur at the normal shock, and the sharp changes appear more forward in real-gas effect considered jet. After the shock, the flow properties tend to be stable, and the differences between the two cases begin to disappear and continue until the far fields. 

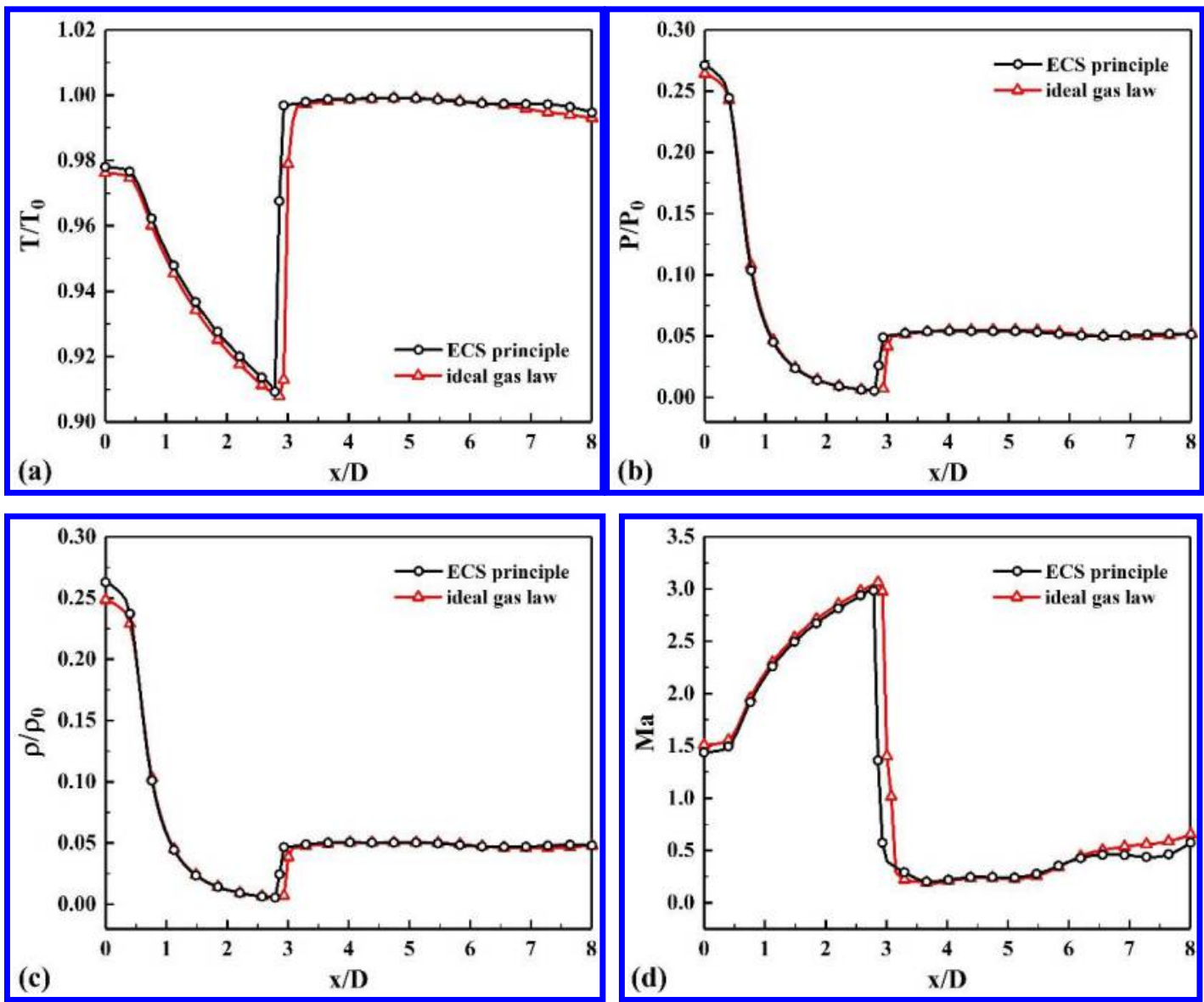

Figure 5. Comparisons of near-fields mean profiles of flow properties between real-gas effects considered (ECS principle) and neglected (ideal gas law) jets along the jet centerline: (a) temperature; (b) pressure; (c) density; (d) Mach number

\section{B. Global flow field characteristics}

Instantaneous fully-developed and real-gas effect considered flow field of temperature at the streamwise section is shown in

Figure 6. Similarity, isolines of kerosene mass fraction, which is the sum of mass fractions of the three components are shown in Figure 7, colored by streamwise velocity. Moreover, the diffusion physics of kerosene characterized by its mass fraction variations is displayed in Figure 8. Distributions of velocity, temperature and mass fraction exactly characterize the momentum, temperature and mass diffusion of kerosene, which determine the mixing potential with air. It also can be found that they explicitly illustrate the jet boundary which can make the instability process of jet clear. In the front part, the jet is almost symmetrical and stabilized without transverse diffusion, and a vortex pair can be observed. In the back of the region, vortex rings are inclining, distorting and breaking up because of the KelvinHelmholtz $(\mathrm{K}-\mathrm{H})$ instability of jet shear layer. Thus, many large-scale coherent structures appear in the flow field, which play a good role in enhancing the mixing of fuel and air. 


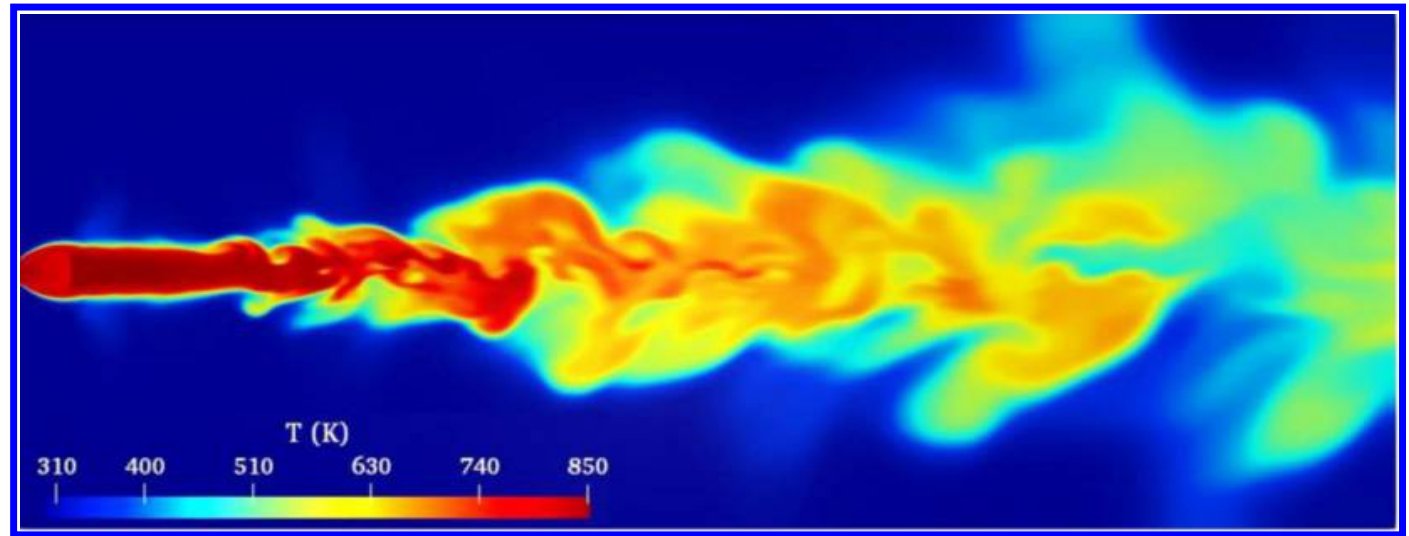

Figure 6. Instantaneous distribution of temperature at the streamwise section $Z=0$ of the fully-developed realgas effect considered jet

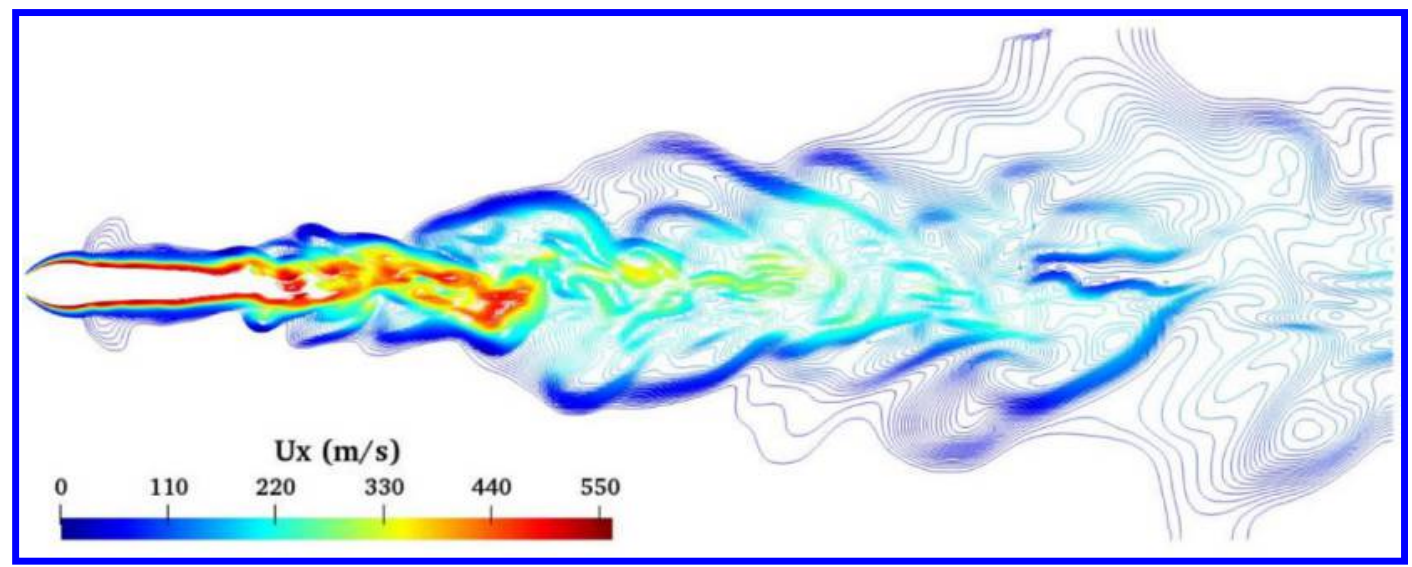

Figure 7. Instantaneous isolines of kerosene mass fraction at the streamwise section $Z=0$, colored by of streamwise velocity $U x$ of fully-developed real-gas effect considered jet

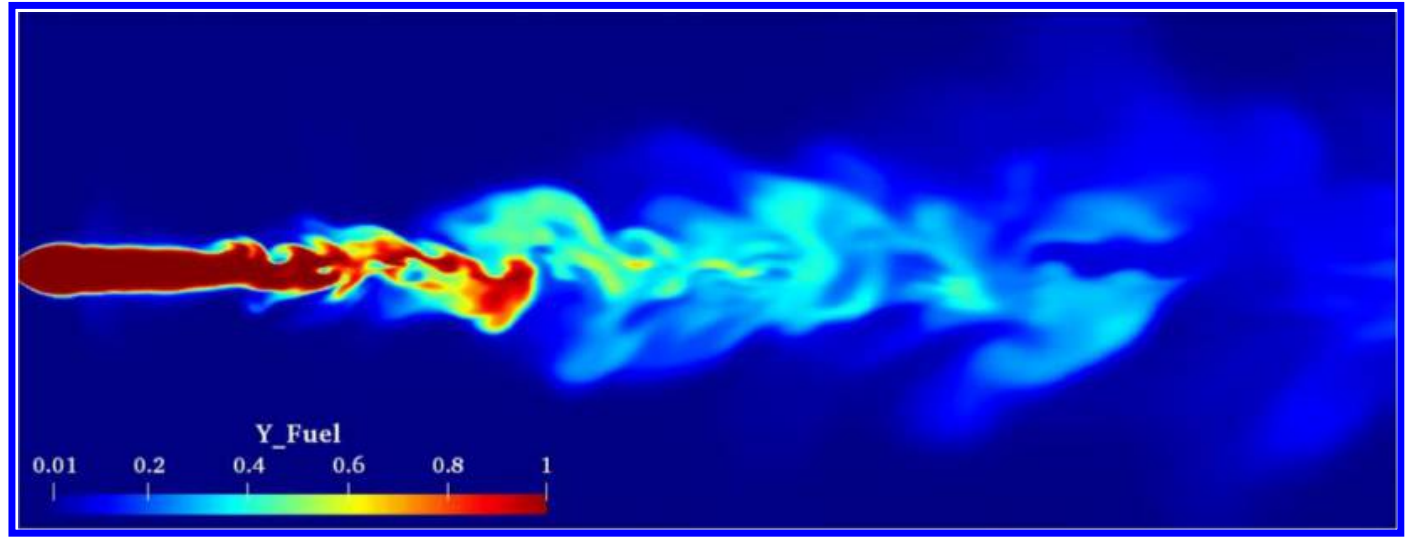

Figure 8. Instantaneous three-component kerosene mass fraction at the streamwise section $Z=0$ of fullydeveloped real-gas effect considered jet

Three-dimensional instantaneous fully-developed and real-gas effect considered isosurfaces of the Q-criterion $\left(\mathrm{Q}=10^{7} \mathrm{~m}^{2} / \mathrm{s}^{2}\right)$ are presented in Figure 9 and are colored by temperature at the same time. Q-criterion is defined as Eq. (5) [34], where $S$ and $\Omega$ are strain rate tensor and rotation rate tensor.

$$
\mathrm{Q}=-\frac{1}{2}\left(\|S\|^{2}-\|\Omega\|^{2}\right)
$$


It is also shown that some annular shear layer forms in near nozzle field, and then circumferential instability occurs leading the whole shear layer to break into small-scale vortex tubes. Many large-scale and three-dimensional streamwise vortices and hairpin vortices can be found around the jet shear layer, which mean the arrival of full turbulence.

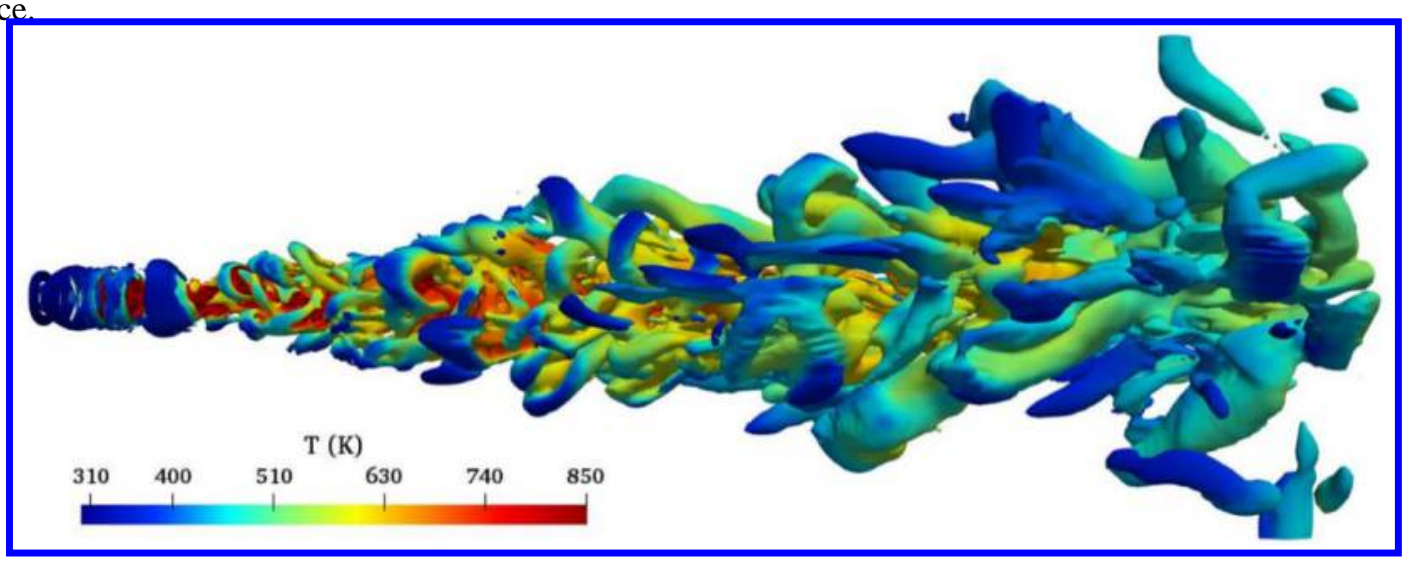

Figure 9. Instantaneous isosurfaces of $Q$-criterion $\left(Q=10^{7} \mathrm{~m}^{2} / \mathrm{s}^{2}\right)$, colored by temperature $T$ of the fullydeveloped real-gas effect considered jet
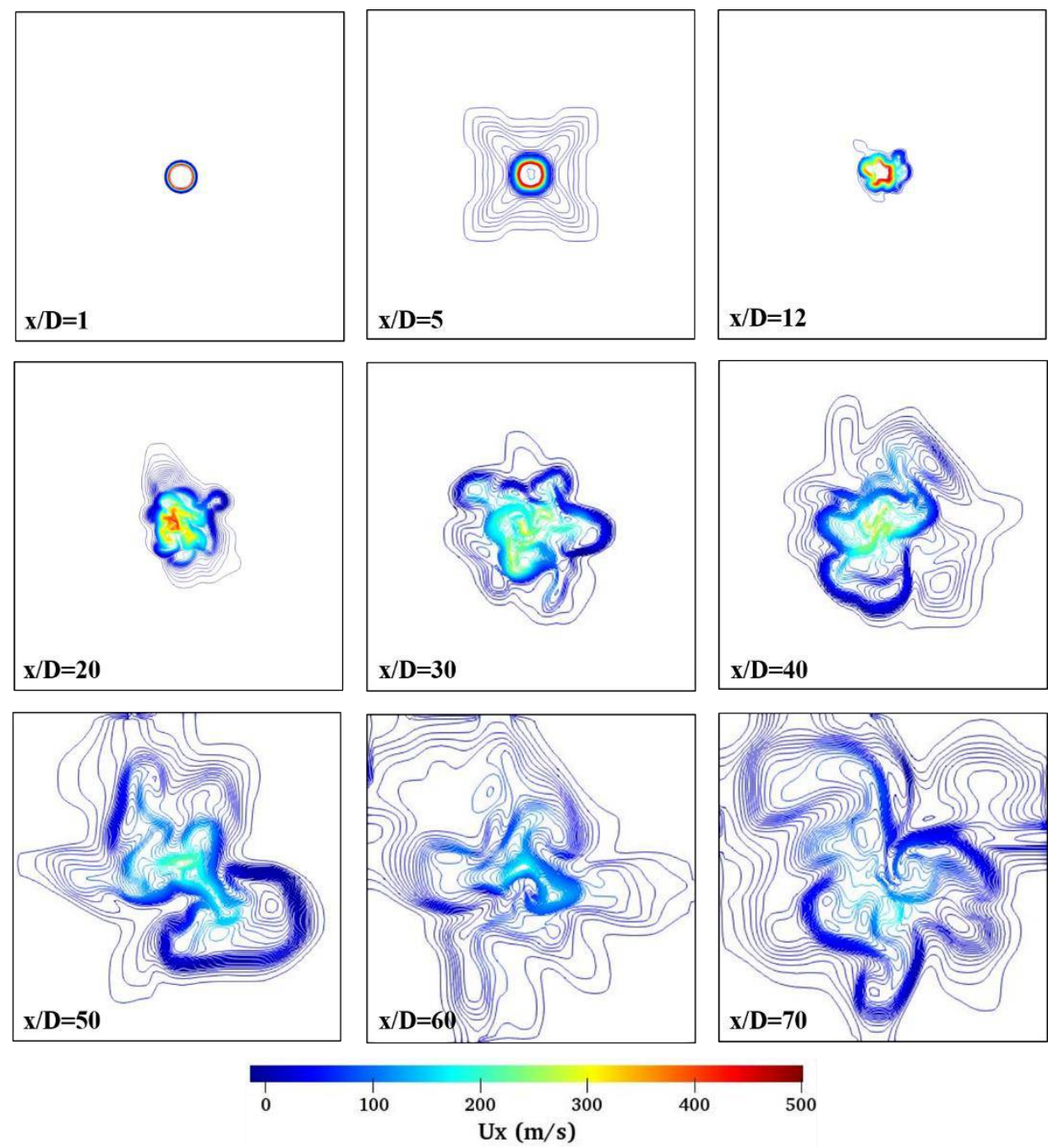

Figure 10. Instantaneous isolines of kerosene mass fraction at transverse sections $x / D=1,5,12,20,30,40,50$, 60,70 , colored by streamwise velocity of the fully-developed real-gas effect considered jet 
Figure 10 displays the isolines of kerosene mass fraction in real-gas effect considered flow field at the transverse sections, which can not only demonstrates the spatial evolution of jet shear layer, especially the circumferential instability, but also shows the mass diffusion process. Isolines are colored by instantaneous streamwise velocity under the same conditions. At cross-section $x / D=1$, the jet boundary is circular. At $x / D=5$, a vortex ring is sharply captured and the jet core is approximately circular. At $\mathrm{x} / \mathrm{D}=12,20$, jet begins to diffuse around, with a tendency of instability. At areas like $\mathrm{x} / \mathrm{D}=30,40$, the circumferential instability of jet shear layer becomes more obvious and the jet structures begin to distort. Moreover, the internal structures also begin to change, which results from the entered vorticity disturbances from the jet boundary. At the downstream sections $x / D=50,60,70$, the velocity of jet is significantly slower, and the area is larger. Furthermore, the increasing small-scale and low-velocity structure of jet shear layer can promote the momentum exchange between the inside and outside the jet, which enhances the mixing of kerosene jet and air.

Note that the qualitative variation tendencies of aerodynamic parameters, vortex structures, evolutionary rules and mass diffusion processes of jet shear layer are quite similar between real-gas effect considered and neglected flow fields. However, quantitatively, the flow properties of them are partly different. For example, differences of global flow-fields mean profiles of kerosene mass faction, which is the sum of mass fractions of the three components along the jet centerline between the two cases are plotted in Figure 11 (a). It is shown that real-gas effect considered jet (use ECS principle) has a little bit shorter potential core and a lower mass fraction along the jet centerline, which partly demonstrates that it has lower streamwise speed and diffuses around faster than real-gas effect neglected jet (use ideal gas law). Moreover, the diffusion areas of the two jets at several transverse sections are presented in Figure 11 (b). The diffusion area is defined as the area covered by the $Y_{\text {Fuel }}=0.05$ contour line. The profiles illustrate that diffusion areas increase rapidly along the main flow direction, corresponding with the decreases of kerosene mass fraction. By contrast, what responses to the differences of kerosene mass fraction between the two jets is that the areas of real-gas effect considered jet are always larger than another.
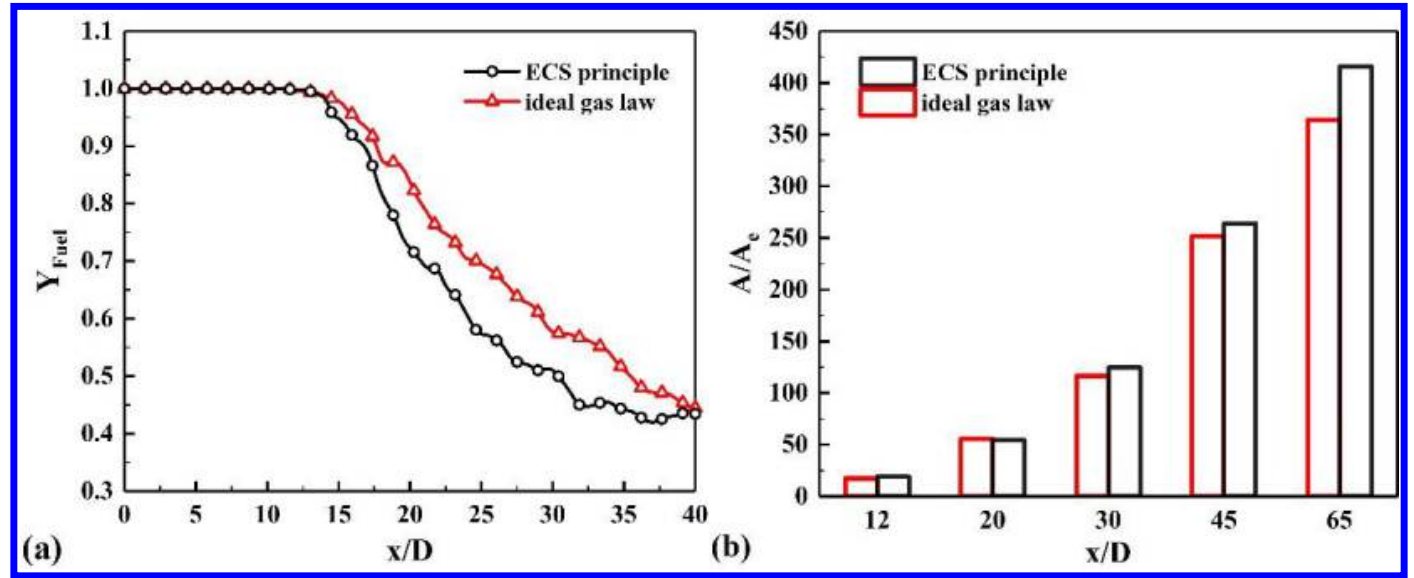

Figure 11. Comparisons of global flow-fields mean profiles of flow properties between real-gas effects considered (ECS principle) and neglected (ideal gas law) jets along the jet centerline: (a) kerosene mass fraction; (b) kerosene diffusion area

\section{Conclusions}

The principle of Extended Corresponding States was applied to model the real-gas effect of supersonic kerosene jet by characterizing the pressure-temperature-density behavior in the three-dimensional large eddy simulation. Two cases including real-gas effect considered jet using the ECS principle and real-gas effect neglected jet using ideal gas law were both set to reveal the penetration, instability, breakup, diffusion and mixing physics in supersonic kerosene jet, and were compared to display the influence of real-gas effect on the jet properties. The modeling results were firstly validated against the measured near nozzle shock structures in experiments, and general good agreements were achieved using the current modeling framework. Then qualitative and quantitative analyses were conducted by the aid of flow parameters distributions in near and global fields. The main conclusions of this study are summarized as follows. 
(1) The local and global flow parameters were presented and analyzed, indicating that the global rules of flow evolutions and developments of the two jets are similar and their turbulence transitions are both characterized by the breakup of vortex pairs, distortion of jet core and generation of vortex structures. Furthermore, the variation tendencies of aerodynamic parameters in near nozzle fields and far fields of them are also alike, and their profiles outline the typical supersonic jet flow fields.

(2) The near nozzle shock structures, e.g., the size of Mach disk and changing profiles of aerodynamic fields, were evaluated quantitatively and comparatively in the two cases. The Mach disk is smaller and closer to nozzle exit in real-gas effect considered jet. And it also has higher temperature, pressure and density and inversely lower Mach number at the nozzle exit and upstream of the normal shock. As for global distributions, the diffusion physics of kerosene jet was characterized by variation rules of its mass fraction and diffusion area, showing that real-gas effect considered kerosene jet has a little bit shorter potential, larger diffusion area and higher diffusion speed than another kerosene jet.

\section{Acknowledgments}

The Project was supported by National Natural Science Foundation of China (Grant No. 11502270) and the Training Program of the Major Research Plan of the National Natural Science Foundation of China (Grant No. 91641110). The authors are also grateful to National Supercomputer Center in Tianjin for providing the computational resource.

\section{References}

1. Fan, X., and Yu, G. "Analysis of thermophysical properties of Daqing RP-3 aviation kerosene," Journal of Propulsion Technology Vol. 27, No. 2, 2006, pp. 187-192.doi: 10.13675/j.cnki.tjjs.2006.02.021

2. Huber, M. L. "NIST Thermophysical Properties of Hydrocarbon Mixtures Database (SUPERTRAPP) V3.1." National Institute of Standards and Technology, 2003.

-3. Petit, X., Ribert, G., Lartigue, G., and Domingo, P. "Large-eddy simulation of supercritical fluid injection," The Journal of Supercritical Fluids Vol. 84, 2013, pp. 61-73.doi: 10.1016/j.supflu.2013.09.011

4. Oefelein, J. C., and Yang, V. "Modeling High-Pressure Mixing and Combustion Processes in Liquid Rocket Engines," Journal of Propulsion \& Power Vol. 14, No. 5, 1998, pp. 843-857.doi: 10.2514/2.5349

-5. Hamzehloo, A., and Aleiferis, P. G. "Large eddy simulation of highly turbulent under-expanded hydrogen and methane jets for gaseous-fuelled internal combustion engines," International Journal of Hydrogen Energy Vol. 39, No. 36, 2014, pp. 21275-21296.doi: 10.1016/j.ijhydene.2014.10.016

6. Bonelli, F., Viggiano, A., and Magi, V. "A Numerical Analysis of Hydrogen Underexpanded Jets Under Real Gas Assumption," Journal of Fluids Engineering-Transactions of the Asme Vol. 135, No. 12, 2013, p. 121101.doi: 10.1115/1.4025253

7. Reid, R. C., Sherwood, T. K., and Street, R. E. "The Properties of Gases and Liquids," Physics Today Vol. 12, No. 4, 1959, pp. 38-40.doi: 10.1063/1.3060771

8. Jiang, C. X., Zhong, F. Q., Fan, X. J., and Yu, G. "Experiment on convective heat transfer of aviation kerosene under supercritical pressures," Journal of Propulsion Technology Vol. 31, No. 2, 2010, pp. 230234.doi: 10.13675/j.cnki.tjjs.2010.02.001

9. Leland, T. W., and Chappelear, P. S. "THE CORRESPONDING STATES PRINCIPLE-A REVIEW OF CURRENT THEORY AND PRACTICE," Industrial \& Engineering Chemistry Vol. 60, No. 7, 1968.doi: 10.1021/ie50703a005

10. Ely, J. F., and Hanley, H. J. M. "Prediction of Transport-Properties .1. Viscosity of Fluids and Mixtures," Industrial \& Engineering Chemistry Fundamentals Vol. 20, No. 4, 1981, pp. 323-332.doi: $10.1021 / \mathrm{I} 100004 \mathrm{a} 004$

-11. Ely, J. F., and Hanley, H. J. M. "Prediction of Transport-Properties .2. Thermal-Conductivity of Pure Fluids and Mixtures," Industrial \& Engineering Chemistry Fundamentals Vol. 22, No. 1, 1983, pp. 90-97.doi: 10.1021/I100009a016

12. Takahashi, S. "PREPARATION OF A GENERALIZED CHART FOR THE DIFFUSION COEFFICIENTS OF GASES AT HIGH PRESSURES," Journal of Chemical Engineering of Japan Vol. 7, No. 6, 1975, pp. 417-420.doi: 10.1252/jcej.7.417

-13. Oefelein, J. C. "Thermophysical characteristics of shear-coaxial LOX-H2 flames at supercritical pressure," Proceedings of the Combustion Institute Vol. 30, No. 2, 2005, pp. 2929-2937.doi:

10.1016/j.proci.2004.08.212 
14. Li, X., Huai, X., Cai, J., Zhong, F., Fan, X., and Guo, Z. "Convective heat transfer characteristics of China RP-3 aviation kerosene at supercritical pressure," Applied Thermal Engineering Vol. 31, 2011, pp. 23602366.doi: 10.1016/j.applthermaleng.2011.03.036

-15. Yu, G., Li, J. G., Chang, X. Y., Chen, L. H., and Sung, C. J. "Fuel injection and flame stabilization in a liquid-kerosene-fueled supersonic combustor," Journal of Propulsion and Power Vol. 19, No. 5, 2003, pp. 885-893.

16. Zhong, F.-Q., Fan, X.-J., Wang, J., Yu, G., and Li, J.-G. "Characteristics of compressible flow of supercritical kerosene," Acta Mechanica Sinica Vol. 28, No. 1, 2012, pp. 8-13.doi: 10.1007/s10409-0120006-X

-17. Yu, G., Li, J. G., Zhang, X. Y., Chen, L. H., and Sung, C. J. "Investigation of Kerosene-Hydrogen Combustion Characteristics in Model Supersonic Combustors," 36th AIAA/ASME/SAE/ASEE Joint Propulsion Conference and Exhibit. Huntsville, Alabama, 2000, pp. AIAA 2000-3620.

18. Yu, G., Li, J. G., Yue, L. J., Zhao, J. R., Zhang, X. Y., Huang, Y., and Sung, C. J. "Characterization of kerosene combustion in supersonic flow using effervescent atomization," 11th AIAA/AAAF International Conference Space Planes and Hypersonic Systems and Technologies. Orleans, France, 2002, pp. AIAA 2002-5225.

19. Yu, G., Li, J. G., Zhao, Z., Chang, X. Y., and Sung, C. J. "Investigation of vaporized kerosene injection in a supersonic model combustor," 12th AIAA International Space Planes and Hypersonic Systems and Technologies. Norfolk, Virginia, 2003, pp. AIAA 2003-6938.

-20. Daniau, E., Bouchez, M., Bounaceur, R., Battin - Leclerc, F., Marquaire, P.-M., and Fournet, R. "Contribution to Scramjet Active Cooling Analysis Using N-dodecane Decomposition Model," 2003.doi: $10.2514 / 6.2003-6920$

21. Dagaut, P. "On the kinetics of hydrocarbons oxidation from natural gas to kerosene and diesel fuel," Physical Chemistry Chemical Physics Vol. 4, No. 11, 2002, pp. 2079-2094.

22. Dagaut, P., Karsenty, F., Dayma, G., Diévart, P., Hadj-Ali, K., Mzé-Ahmed, A., Braun-Unkhoff, M., Herzler, J., Kathrotia, T., Kick, T., Naumann, C., Riedel, U., and Thomas, L. "Experimental and detailed kinetic model for the oxidation of a Gas to Liquid (GtL) jet fuel," Combustion and Flame Vol. 161, No. 3, 2014, pp. 835-847.doi: 10.1016/j.combustflame.2013.08.015

23. Yoshizawa, A. "Statistical theory for compressible turbulent shear flows, with the application to subgrid modeling," Physics of Fluids Vol. 29, No. 7, 1986, p. 2152.doi: 10.1063/1.865552

24. Li, X., Yao, W., and Fan, X. "Large-Eddy Simulation of Time Evolution and Instability of Highly Underexpanded Sonic Jets," AIAA Journal Vol. 54, No. 10, 2016, pp. 3191-3211.doi: 10.2514/1.J054689

25. Li, X., Wu, K., Yao, W., and Fan, X. "A comparative study of highly underexpanded nitrogen and hydrogen jets using large eddy simulation," International Journal of Hydrogen Energy Vol. 41, No. 9 , 2016, pp. 5151-5161.doi: 10.1016/j.ijhydene.2016.01.120

-26. Yao, W., Wang, J., Lu, Y., Li, X., and Fan, X. "Full-scale Detached Eddy Simulation of kerosene fueled scramjet combustor based on skeletal mechanism," 20th AIAA International Space Planes and Hypersonic Systems and Technologies Conference. American Institute of Aeronautics and Astronautics, 2015.

27. Yao, W., Lu, Y., Li, X., Wang, J., and Fan, X. "Detached Eddy Simulation of a high-Ma regenerativecooled scramjet combustor based on skeletal kerosene mechanism," 52nd AIAA/SAE/ASEE Joint Propulsion Conference. American Institute of Aeronautics and Astronautics, 2016.

-28. Yao, W., Yuan, Y., Li, X., Wang, J., and Fan, X. "A comparative study of elliptical and round scramjet combustors by Improved Delayed Detached Eddy Simulation," Aiaa International Space Planes and Hypersonics Technologies Conference. 2017.

29. OpenCFD Ltd. Openfoam 3.0.1. http://www.openfoam.org/, 2015

30. Chase, M. W. JANAF thermochemical tables: GOVERNMENT PRINTING OFFICE, 1986.

-31. Kurganov, A., and Tadmor, E. "New High-Resolution Central Schemes for Nonlinear Conservation Laws and Convection-Diffusion Equations," Journal of Computational Physics Vol. 160, No. 1, 2000, pp. 241282.doi: $10.1006 /$ jcph.2000.6459

-32. Vuorinen, V., Larmi, M., Schlatter, P., Fuchs, L., and Boersma, B. J. "A low-dissipative, scale-selective discretization scheme for the Navier-Stokes equations," Computers \& Fluids Vol. 70, 2012, pp. 195205.doi: 10.1016/j.compfluid.2012.09.022

-33. Crist, S., Glass, D. R., and Sherman, P. M. "Study of the highly underexpanded sonic jet," AIAA Journal Vol. 4, No. 1, 1966, pp. 68-71.doi: 10.2514/3.3386

34. Jeong, J., and Hussain, F. "On the identification of a vortex," Journal of Fluid Mechanics Vol. 285, No. -1, 2006, p. 69.doi: $10.1017 / \mathrm{s} 0022112095000462$

12

American Institute of Aeronautics and Astronautics 